\title{
Inventaire commenté des Thysanoptères de Nouvelle-Calédonie
} Jean-Paul Bournier, Laurence A. Mound

\section{Abstract}

Thysanoptera from New-Caledonia : an annoted check list. Sixty-eight species in 44 genera are listed, 26 species and 15 genera for the first time in New-Caledonia. For each of them short data of biology and geographic distribution are given, as well as records of samples collected in the island.

\section{Résumé}

Soixante-huit espèces dans 44 genres sont inventoriées dont 26 espèces et 15 genres sont nouveaux pour la NouvelleCalédonie. Pour chacune des espèces quelques brefs éléments de leur biologie et de leur distribution géographique sont présentés, ainsi que les références du matériel collecté sur l'île.

\section{Citer ce document / Cite this document :}

Bournier Jean-Paul, Mound Laurence A. Inventaire commenté des Thysanoptères de Nouvelle-Calédonie. In: Bulletin de la Société entomologique de France, volume 105 (3), août 2000. pp. 231-240;

https://www.persee.fr/doc/bsef_0037-928x_2000_num_105_3_16666

Fichier pdf généré le 30/09/2019 


\title{
Inventaire commenté des Thysanoptères de Nouvelle-Calédonie
}

\author{
par Jean-Paul BouRNIER* et Laurence A. MOUND** \\ * CIRAD-AMIS ; Protection des Cultures, Entomologie tropicale, TA 40/02, F- 34398 Montpellier cedex 5 \\ ** CSIRO Entomology, GPO Box 1700, Canberra City, Australia
}

Résumé. - Soixante-huit espèces dans 44 genres sont inventoriées dont 26 espèces et 15 genres sont nouveaux pour la Nouvelle-Calédonie. Pour chacune des espèces quelques brefs éléments de leur biologie et de leur distribution géographique sont présentés, ainsi que les références du matériel collecté sur l'île.

Summary. - Thysanoptera from New-Caledonia : an annoted check list. Sixty-eight species in 44 genera are listed, 26 species and 15 genera for the first time in New-Caledonia. For each of them short data of biology and geographic distribution are given, as well as records of samples collected in the island.

Key words. - Thysanoptera, Faunistic, New-Caledonia.

Grande-Terre, la plus étendue des îles du territoire de Nouvelle-Calédonie, mesure $400 \mathrm{~km}$ de long et 50 à $70 \mathrm{~km}$ de large. Située juste au nord du tropique du Capricorne, elle présente une flore remarquablement diversifiée pour sa superficie, avec plus de 2600 espèces végétales dans environ 600 genres (GUILLAUMIN, 1948). Cependant l'évaluation de la richesse de la faune entomologique en relation avec la diversité botanique n'a jusqu'à présent été que bien peu étudiée (CHAZEAU, 1995). Malgré cela, la faune des Thysanoptères de NouvelleCalédonie est relativement documentée en comparaison de celle d'autres territoires de Mélanésie ou de Micronésie, cette remarque ayant pour but de montrer combien cet ordre d'insectes a été peu étudié dans les îles du Pacifique.

Les publications abordant la faune des Thysanoptères de la vaste zone s'étendant de Nouvelle-Guinée à la Polynésie sont relativement rares. BAGNALL décrit quelques espèces de Nouvelle-Guinée (1908) et des Samoa et Tonga (1928). MoulToN décrit plusieurs espèces de Polynésie (1939; voir aussi Moulton \& Steinweden, 1932, 1933), des Fidji (1944), de Nouvelle-Guinée et de Nouvelle-Bretagne (1940, 1947). SAKIMURA (1969) décrit trois espèces des Fidji, Tonga et Nouvelle-Calédonie, et MouND (1970) une série de taxa des îles Salomon. Il n'existe cependant pas d'inventaire général des Thysanoptères originaires de cette région ; il faut noter que MOUND (1996) et MOUND \& WALKER $(1982,1986)$ ont réalisé ce genre d'étude pour la faune des Thysanoptères d'Australie et de Nouvelle-Zélande.

Si les informations manquent pour les espèces originaires de la faune du Pacifique, on trouve au contraire un grand nombre de données pour de nombreuses espèces cosmopolites largement répandues dans la plupart des îles du Pacifique. La dispersion de ces espèces, associées, pour la plupart, aux plantes cultivées, est sans conteste la conséquence des activités humaines dans cette région, mais aussi, il y a encore peu de temps, due au manque de mesures de quarantaine efficaces entre les diverses îles. MOUND \& WALKER (1987) inventorient plus de 30 espèces cosmopolites aux Fidji, au Kiribati (îles Gilbert) et Vanuatu; Mound (1999a) liste 34 espèces de l'île Lord Howe, pour la plupart elles aussi cosmopolites.

La réalisation de l'inventaire qui fait l'objet de cette publication est la conséquence d'une visite en Nouvelle-Calédonie en fin décembre 1998 et qui a permis la découverte d'une vingtaine d'espèces pantropicales jusqu'alors inconnues sur ce territoire.

Le premier Thysanoptère signalé de Nouvelle-Calédonie a été décrit il y a 92 ans par BAGNALL (1908); cependant, la première et importante contribution à la connaissance de la faune des Thrips de cette île est l'œuvre de BIANCHI $(1945,1952)$, qui décrit 10 espèces et 
en cite 13 autres déjà connues. Un quart de siècle plus tard, SAKIMURA (1969) décrit une nouvelle espèce. Par la suite, cinq autres nouvelles espèces sont décrites par Alexandre BOURNIER $(1975,1993)$ puis quatre nouvelles espèces et un genre nouveau (BOURNILR J.-P., 1997). Ces sept publications, couvrant une période de 89 ans, totalisent 34 espèces.

Cependant, des collectes effectuées par nébulisation d'arbres dans les forêts primaires de l'île (BOURNIER, 1997) laissent à penser que de nombreuses espèces restent à décrire; de même les récentes collectes, mentionnées dans la liste ci-dessous, et qui ont été réalisées, pour la plupart, sur une végétation herbacée basse, tendent à confirmer la richesse de cette faune.

En dépit du peu d'informations, et des nombreuses espèces qui restent à décrire, il semble certain que la Nouvelle-Calédonie possède une faune endémique de Thysanoptères très intéressante. Chez les Aeolothripidae on note une espèce endémique du genre Desmidothrips ; chez les Thripidae, trois espèces du genre cosmopolite Thrips paraissent être endémiques à l'île; un nouveau genre et une nouvelle espèce de Dendrothripinae, Filicopsothrips wellsae Mound, récolté sur fougères arborescentes, ont été récemment décrits (MOUND, 1999b). Chez les Phlaeothripidae phyllophages, le genre Teuchothrips, dont les espèces induisent des déformations foliaires ou des galles sur différentes plantes, comprend plusieurs espèces dont certaines ne sont pas encore décrites. Chez les Phlaeothripidae mycophages, le groupe le plus diversifié des Thysanoptères et pour lequel bien peu est connu, les chances d'endémisme sont moins évidentes, bien que certaines espèces ne semblent pas être cosmopolites. Les relations entre la faune des Thrips de Nouvelle-Calédonie et celle des régions avoisinantes ne sont pas aisément prévisibles; cependant Desmidothrips est aussi connu de NouvelleZélande, une seconde espèce d'Ensiferothrips a récemment été décrite de l'île Lord Howe (MOUND, 1999a), et Teuchothrips comprend de nombreuses espèces voisines dans l'est australien; en dépit d'investigations considérables, il n'a pas été possible de trouver des espèces gallicoles sur les Acacia sp. autochtones, alors que l'on rencontre de nombreuses espèces en Australie (MOUND, 1996).

*: les espèces marquées d'un astérisque sont signalées pour la première fois de Nouvelle-Calédonic.

\section{MEROTHRIPIDAE}

Merothrips floridensis Watson, 1927*

Biologie : espèce mycophage sur bois mort.

Distribution: très répandu dans le monde tropical et sub-tropical.

Matériel collecté : 1 ๆ, sur branches mortes, cascade Farino, 20.XII.1998.

\section{AEOLOTHRIPIDAE}

Desmidothrips inauditus (Bianchi, 1945)

Biologie: floricole et vraisemblablement prédateur facultatif d'autres Thrips.

Distribution: Nouvelle-Calédonie (D. walkerae Mound, de Nouvelle-Zélande, est très voisin).

Matériel collecté : mont Nondoué, vallée de Yahoué, Oua Tom, et collines autour de Nouméa (BiAN(III. 1945, BOURNIER, 1997).

Franklinothrips vespiformis (Crawford, 1909)*

Biologie : prédateur d'autres Thrips, sur feuilles.

Distribution: trc̀s rćpandu dans la rćgion nćutropicale, Iridji. Thaïlandc, îlc de la Rćunion.

Matériel collecté: 1 , 1 larve sur fleurs de Leucaena. Pouembout, 22.XII.1998.

\section{THRIPIDAE}

Anaphothrips sudanensis Trybom, 1911

Biologie: sur feuilles de plantes herbacées, y compris Zea maïs et Saccharum.

Distribution: très répandu cans les régions tropicales et sub-tropicales.

Matériel collecté: Népoui et Nouméa, sur plantes herbacées et canne à sucre (BıA.v(III. 1945). 
Anaphothrips swezeyi Moulton, 1928

Biologie : sur feuilles de plantes herbacées, y compris Saccharum.

Distribution: Hawaï, Nouvelle-Calédonie.

Matériel collecté: Népoui et Nouméa (BIANCHI, 1945); plusieurs $\&$ sur plantes herbacées, route du Cap, Bourail, 22.XII. 1998.

Arorathrips mexicanus (Crawford, 1909)*

Biologie : sur fleurs de plantes herbacées, avec une seule larve par fleur.

Distribution : très largement distribué dans les zones tropicales et subtropicales.

Matériel collecté: $q$ \& et $\delta$ ò sur piantes herhacées, route du Cap. Bourail. 21.XII.1998; Houailou. 26.XII.1998.

\section{Bolacothrips pulcher Girault, 1929*}

Biologie: sur plantes herbacées.

Distribution: zones humides du nord Australien.

Matériel collecté : $q q$ et ơ ô sur plantes herbacées, route du Cap, Bourail, 22.XII.1998.

\section{Bregmatothrips saccharicolus (Bianchi, 1945)}

Biologie: sur feuilles de graminées y compris canne à sucre.

Distribution: est Australien, Nouvelle-Calédonie.

Matériel collecté : 59 . Nouméa (BIANCHI, 1945); $q$ q et ơ ơ sur herbes, route du Cap, Bourail, 22.XII. 1998 ; sur herbes, Parc-Rivière-Bleue, 19.XII.1998.

\section{Caprithrips insularis Beshear, 1975*}

Biologie : sur feuilles de graminées.

Distribution: USA (Georgie), Trinidad, Surinam. Kiribati

Matériel collecté: $\subsetneq \subsetneq$ sur herbes, au jord de la route du Cap, $25 \mathrm{~km}$ au nord de Bourail, 22.XIl.1998.

Caprithrips orientalis Bhatti, 1973*

Biologie : sur feuilles de plantes herbacćes.

Distribution: Inde, Fidji.

Matériel collecté: $q \uparrow$ sur herbes au bord de la route du Cap, à $25 \mathrm{~km}$ au nord de Bourail, 22.XII.1998.

Chirothrips ah Girault, 1929* (identifié à tort par BIANCHI, 1945 comme C. aculeatus)

Biologie : sur fleurs de plantes herbacées.

Distribution: est de l'Australie, Nouvelle-Guinée, Afrique du Sud.

Matériel collecté: Nouméa, sur canne à sucre, herbe de Johnson et fleurs de Lantana sp. (BIANCHI. 1945); 1 sur herbes au bord de la route du Cap, à $25 \mathrm{~km}$ au nord de Bourail, 22.XII.1998.

\section{Dendrothripoides innoxius (Karny, 1914)*}

Biologie : communément trouvé sur feuilles de diverses espèces d'Ipomoea.

Distribution: très largement distribué dans les régions tropicales.

Matériel collecté: $3 \uparrow$ sur feuilles d'arbustes, $20 \mathrm{~km}$ à l'est de Pouembout, 22.XII.1998.

\section{Dendrothrips reticulatus Bournier, 1997}

Biologie : les espèces de ce genre vivent toutes sur les feuilles d'arbustes ou d'arbres.

Distribution: Nouvelle-Calédonie.

Matériel collecté: $13 \%, 1 \delta^{\star}$, Mont-Nondoué (BOURNIER. 1997):1\% sur feuilles de Psidium, $20 \mathrm{~km}$ à l'ouest de Thio. 28.XII.1998.

\section{Ensiferothrips primus Bianchi, 1945}

Biologie : sur feuilles de Malaisia scandens (Moraceae).

Distribution: est de l'Australie, Nouvelle-Calédonie.

Matériel collecté: 2\%. Nouméa (Bianchi. 1945); 89 , Mont-Nondoué et Pindai (Bournier, 1997): 49.21 ठ et larves sur feuilles de Malaisia scandens, $20 \mathrm{~km}$ à l'est de Pouembout 22.XII.1998:

1 9 . par battage sur végétation diverse. à Hienghène. 25.XII.1998. 
Filicopsothrips wellsae Mound, 1999b

Biologie : sur les frondes de Dicksonia sp. et certainement d'autres fougères arborescentes.

Distribution: Nouvelle-Calédonie.

Matériel collecté : Rivière-Bleue (Mound, 1999b).

Frankliniella occidentalis (Pergande, 1895)*

Biologie: se nourrit et se reproduit sur fleurs et feuilles de nombreuses plantes cultivees (en particulier sur cultures maraîchères) et sauvages; il est responsable de graves dégâts directs, mais aussi indirects en transmettant le Tomato Spotted Wilt Virus: parfois prédateur d'acariens.

Distribution: depuis une quizaine d'années il a envahi l'ensemble des continents aussi bien en zoncs tropicales que tempérées; sous scrre dans les régions plus septentrionales.

Matériel collecté: 14 \&, 3 ò sur rosier à Port-La-Guerre, 20.X.1994 (leg. P. Dully).

Frankliniella schultzei (Trybom, 1910)*

Biologie: se nourrit des tissus des fleurs et des feuilles de nombreux végétaux ; parfois prédateur d'Acariens. Distribution : très largement distribué dans toutes les régions tropicales.

Matériel collecté : 19 par battage sur végétation diverse, cascade de Farino, 20.XII.1998; 19 sur lleurs jaunes de Lupinus sp., $25 \mathrm{~km}$ à l'est de Pouembout, 22.XII.1998; $q$ et ơ sur fleurs blanches de Solanum? mauriteanum, $30 \mathrm{~km}$ à l'est de Pouembout, 23.XII.1998; $q$ sur fleurs blanches d'une Polygonaceae indéterminée et sur feuilles de Mimosa invisa, $15 \mathrm{~km}$ à l'est de Voh, 22.XII.1998: 9 sur fleurs blanches d'une Apiaceae indéterminée, La-Foa, 27.XII.1998. Tous ces spécimens étaient des formes claires de cette espèce.

Heliothrips haemorrhoidalis (Bouché, 1833)

Biologie : phyllophage sur un grand nombre de plantes.

Distribution: sur l'ensemble des continents; en zones tempérées cette espèce se rencontre dans les serres, d'où son nom "Thrips des serres".

Matériel collecté: Mont-Nondoué, Rivière-Bleue (BOuRnifr, 1997); $q q$ récoltées par battage. cascade de Farino, 20.XII.1998.

Hercinothrips bicinctus (Bagnall, 1919)*

Biologie: phyllophage; se multiplie sur un grand nombre de plantes ; parfois déprédateur du bananier.

Distribution: très largement rencontré dans les zones sub-tropicales.

Matériel collecté : $\$ \uparrow$ et larves abondantes sur feuilles de Passiflora sp., $20 \mathrm{~km}$ à l'ouest de Thio. 28.XII.1998.

Megalurothrips usitatus (Baignall, 1913)*

Biologie: se nourrit et se reproduit dans les fleurs de diverses légumineuses.

Distribution: très largement répandu de l'Inde aux îles Fidji y compris l'est de l'Australie.

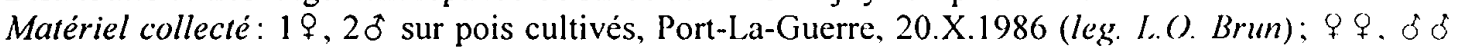
et larves dans les fleurs jaunes de ? Lupinus sp., $25 \mathrm{~km}$ à l'est de Pouembout. 22.XII.1998, ainsi que sur la même plante hôte à Hienghène, 20.XII.1998.

Microcephalothrips abdominalis (Crawford, 1910)*

Biologie: se multiplie dans les fleurs de composées, en particulier sur Helianthus.

Distribution : très largement répandu dans les zones tropicales et sub-tropicales, y compris l'Australic et la Nouvelle-Zélande.

Matériel collecté : 1 récoltée par battage sur végétation diverse. cascade de Farir.o, 20.XII.1998.

Oxythrips festivus Bianchi, 1952

Biologie: inconnue.

Distribution: Nouvelle-Calédonie.

Matériel collecté : Bois-du-Sud (BIANCHI, 1952).

\section{Pezothrips kellyanus (Bagnall, 1916)}

Biologie: se reproduit dans diverses fleurs blanches odoriférantes, en particulier ('itrus et .Jasminum. Distribution: est et sud de l'Australie, Nouvelle-Zélande, Nouvelle-Calédonie, Grèce, sud de l'Italie. Matériel collecté: Nouméa et Hienghène, dans les fleurs de Cerbera (Apocynaceae) (BIANcHI. 1945); $\&$ dans les fleurs de Thevetia perwviana (Apocynaceae), plage de Poé, 21.XII.1998. 
Phibalothrips longiceps (Karny, 1913)*

Biologie: se reproduit sur diverses plantes basses.

Distribution: est de l'Australie. Nouvelle-Guinée. Nouvelle-Angleterre, Fidji.

Matériel collecté : 9 sur herbes. cascade de Farino, 20.XII.1998; 1 s sur herbes, $10 \mathrm{~km}$ à l'ouest de Ilouailou. 26.XII.1998.

Plesiothrips perplexus (Beach, 1896)*

Biologie : se reproduit dans les fleurs de plantes herbacées.

Distribution : largement répandu dans les zones tropicales, ainsi qu'en Nouvelle-Zélande.

Matériel collecté: 1 \% sur herbes le long de la route du Cap, à $25 \mathrm{~km}$ au nord de Bourail, 22.XI1.1998.

Rhamphothrips pandens Sakimura, 1983*

Biologie: inconnue, mais vraisemblablement phyllophage.

Distribution: dans toutes les zones tropicales.

Matériel collecté : $5 \%$. sur feuilles d'arbustes, $20 \mathrm{~km}$ à l'est de Pouembout; $q$ sur herbes le long de la route du Cap à $25 \mathrm{~km}$ au nord de Bourail, 22.XII. 1998.

\section{Scirtothrips albomaculatus Bianchi, 1945}

Biologie : phyllophage et apparemment sur un grand nombre de plantes.

Distribution: est de l'Australie. île Lord Howe (MOUND, 1999 a), Nouvelle-Calédonie.

Matériel collecté: Nouméa (BIANCHI, 1945); Rivière-Bleue (BOURNIER, 1997); $\$ q$, ơ of et larves sur fleurs et feuilles d'Acacia spirorbis, plage de Poé, 20.XII.1998, sur feuilles d'Acacia nilotica. $30 \mathrm{~km}$ à l'est de Pouembout, 23.XII.1998, et sur Mimosa pudica, 40 km à l'est de Koné, 24.XII.1998.

Scirtothrips australiae Hood, 1919

Biologie : phyllophage. mais la plante hôte reste inconnue.

Distribution: est de l'Australie. Nouvelle-Calédonie.

Matériel collecté: Rivière-Bleue (BOURNIER, 1997).

Scirtothrips dorsalis Hood.1919*

Biologie: phyllophage. connu comme important ravageur de plusieurs cultures.

Distribution: largement répandu dans toutes les zones tropicales.

Matériel collecté: $q 9$ et ơ ơ sur feuilles de Leucaena, Pouembout, 22.XII.1998; $q$ \& sur feuilles de Mimosa invisa, $15 \mathrm{~km}$ à l'est de Voh, 23.XII.1998.

\section{Selenothrips rubrocinctus (Giard, 1901)}

Biologie: phyllophage, se reproduit à la face inférieure des feuilles de nombreuses plantes et diverses cultures en particulier sur cacaoyer, d'où sa dénomination anglophone "The cocoa Thrips".

Distribution: tropical et sub-tropical sur tous les continents.

Matériel collecté: Nouméa (BIANCHI, 1945); 99 sur Ixora sp., La Foa, XII.1998 (leg. J. Mestre); adultes et larves sur feuilles de jeunes plants de Cleistocalyx (Myrtaceae), route du Cap, $25 \mathrm{~km}$ au nord de Bourail, 22.XII.1998.

Thrips bianchii Sakimura, 1969

Biologie : vraisemblablement floricole.

Distribution: Nouvelle-Calédonie.

Matériel collecté : Nouméa, Hienghène, Thio, Oua Tom (BiAnCHI, 1945; cité à tort comme T. eticollis Bagn.) (Sakimura, 1969): $९$ Q el ô ô sur fleurs blanches de Psychotria (Rubiaceae), plage de Poé, 20.XII.1998.

Thrips imaginis Bagnall, 1926

Biologie : floricole et phyllophage sur diverses plantes.

Distribution: Australie. Nouvelle-Calédonie.

Matériel collecté: Nouméa (BIANCHI, 1945); $q$ sur fleurs de clémentinier, Pocquereux le 18.VIII.1997 (leg. J.P. Deguine) : $q \uparrow$ et ơ ơ sur fleurs et feuilles d'Acacia spirorbis, plage de Poé, 20.XII.1998: 19 sur herbes au bord de la route du Cap à $25 \mathrm{~km}$ au nord de Bourail, 22.XII.1998.

Thrips insignis (Bianchi, 1945)

Biologie: floricole.

Distrihution: Nouvelle-Calédonie. 
Matériel collecté: vallée de Yahoué (BIANCHI, 1945);23,19 sur fleurs d'Eriaxis rigicla (Orchidaceą). parc de Rivière-Bleue, 19.XII.1998.

\section{Thrips maculicollis (Hood, 1918)*}

Biologie : floricole.

Distribution: est de l'Australie.

Matériel collecté: 9 ㅇ. $\delta$ o t larves sur fleurs blanches de Fagraea schlechteri, cascade de lanino. 22.XII.1998; 1 q sur fleurs blanches (Cunoniaceac), plage de Poé, 22.XII.1998.

Thrips novocaledonensis (Bianchi, 1945)

Biologie: floricole sur différentes espèces végétales.

Distribution: Nouvelle-Calédonie.

Matériel collecté: Nouméa, vallée de Yahoué, La-Foa (BIANCHI, 1945); 79 sur lleurs de clémentinier. Pocquereux le 18.VIII.1997 (leg. J.P. Deguine); nombreuses 9, $7 \delta$ sur Macadamia. l.a-Foa. 7.X. 1998 (leg. J. Mestre); nombreuses $\$, \delta$ et larves sur fleurs d'Albizia lebbeck à Bourail. 7.X.1998 (ickem): nombreuses $q$ et $\delta$ sur fleurs d'Eriaxis rigida (Orchidaceae), parc de Rivière-Bleue. 19.XII.1998: nombreuses $\$$, of et larves sur fleurs blanches de Fagraea schlechteri, cascade de Farino, 20.XII. 1908: nombreuses $q$ et $\delta$ sur fleurs blanches d'une Cunoniaceae non identifiée, plage de Poé, 21.XII.1998.

Thrips orientalis, (Bagnall, 1915)*

Biologie : floricole sur différentes espèces végétales, en particulier Jasminum et Gardenia.

Distribution: très largement connu de l'Inde au Japon et Tahiti, ainsi que des Caraibes.

Matériel collecté : nombreux 5, $q$ et larves, Bourail, sur fleurs de Jasminum, X.1998 (leg. J. Mes/re).

Thrips palmi Karny, 1925

Biologie : espèce particulièrement polyphage sur flcurs et feuilles, responsable de sévères dégâts.

Distribution: très largement connu de la plupart des régions tropicales et sub-tropicales; vraisemblablement introduit en Nouvelle-Calédonie en 1980.

Matériel collecté : nombreuses $q$ sur Macroptilium arthropurpureus et Nicandra physalodes à Païta. 22.VII.1986 (leg. L.O. Brun); nombreuses $q$ et ot sur Ageratum conyzioides à Katiramona. 22.VII. 1986 (idem); nombreuses $\&$ sur Grassocephalum crepidioides. La-Coulée. 7.VIII.1980 (idem): nombreuses $q$ et $\delta$ sur Chenopodium murale, Dumbéa. 21.VIII.1986 (idem): nombreuses $q$ sur Solanum nigrum, Païta, 30.IX.1986 (idem); nombreuses \& sur Solamum melongena. Ilienghène. 23.X.1993 (leg. D. Bordat); nombreuses $\&$ et $\delta$ sur Cucumis sativa, Boulouparis, 20.X.1993 (leg. D. Borda:); + sur fleurs de Gomphrena sp. (Amaranthaceae) $40 \mathrm{~km}$ à l'est de Konć. 24.X11.1998: $\$$ sur fleurs d'une Apiaceae non identifiće, La-Foa, 27.XII.1998.

Thrips simplex (Morison, 1930)

Biologie : appelé "Thrips du glaïeul", cette espèce est responsable de sévères dégâts sur les féuilles. fleurs et bulbes de cette plante ; on la rencontre aussi exceptionnellement sur (Killet. Iris. Narcisse.

Distribution: originaire vraisemblablement d'Afrique du Sud, elle a été rapidement disséminée dans le monde entier grâce au commerce des bulbes de glaïeul.

Matériel collecté : Couss (1953).

Thrips tabaci Lindeman, 1889

Biologie : le "Thrips de l'oignon" vit sur un très grand nombre de végétaux sur lesquels, il est souvent responsable d'important dégâts; parfois vecteur du T.S.W.V; parfois prédateur d'Acariens.

Distribution: dans le monde entier. mais peu commun dans les zones tropicales humides.

Matériel collecté: sur Oignon, Nakety (BIANCHI, 1945); nombreuses $q$ et of sur Poireau. Pouembout. 29.VII. 1986 (leg. L.O. Brun).

Thrips vitticornis Karny, 1922*

Biologie : se rencontre sur différentes fleurs.

Distribution: de l'Inde aux îles Fidji.

Matériel collecté $: 9$ et ơ sur fleurs de Canavalia, $20 \mathrm{~km}$ à l'est de Pouembout. 22.XII.1998: 9 et sur fleurs de Canavalia, Hienghène, 25.XII.1998: $q$ et $\delta$ sur fleurs de Vigne, $30 \mathrm{~km}$ a l'ouest de Houailou, 26.XII.1998. 


\section{PhI.AEOTHRIPIDAE IDOLOTHRIPINAE}

Egchocephalothrips monstrosus (Bagnall, 1909)

Biologie : mycophage.

Distribution: Nouvelle-Calédonie.

Matériel collecté : mont Kogin (Bagnall, 1909).

Ethirothrips adventor Bianchi, 1945

Biologie: se nourrit de spores de champignons sur branches mortes.

Distribution: Nouvelle-Calédonie.

Matériel collecté : Nouméa (BIANCHI, 1945).

Ethirothrips stenomelas (Walker, 1859) = adventor Bianchi, 1945

Biologie : se nourrit de spores de champignons sur branches mortes.

Distribution: largement répandu dans les régions tropicales, y compris l'est de l'Australie.

Matériel collecté: Nouméa (BIANCHI, 1952).

Gastrothrips acuticornis Hood, 1925, = noumeae Bianchi, 1945

Biologie: se nourrit de spores de champignons sur branches mortes.

Distribution: largement répandu dans les zones tropicales.

Matériel collecté : Nouméa (BIANCHI, 1945).

Neosmerinthothrips longisetis Bournier, 1997

Biologie: se nourrit aux dépens de spores de champignons sur branches mortes.

Distribution: Nouvelle-Calédonie.

Matériel collecté: Rivière-Bleue (BOURNIER, 1997); 2 sur branches mortes de Ricinus, à $15 \mathrm{~km}$ à l'est de Voh, 23.XII.1998.

Nesothrips lativentris (Karny, 1913)*

Biologie: se nourrit aux dépens de spores de champignons sur branches mortes et feuilles mortes de palmier. Distribution : largement répandu dans les zones tropicales.

Matériel collecté : 1 \%, 1 ơ sur branches mortes, 40 km à l'est de Koné, 24.XII.1998.

\section{Nesothrips propinquus (Bagnall, 1916)}

Biologie : se nourrit aux dépens de spores de champignon, à la base de touffes d'herbes

Distribution: Nouvelle-Zélande, Australie, Nouvelle-Calédonie, Afrique du Sud, Açores.

Matériel collecté: Nouméa (BIANCFi, 1945); $q$ et ô dans herbes au bord de la route du Cap, $25 \mathrm{~km}$ au nord de Bourail, 22.XII.1998.

\section{PhLAEOTHRIPIDAE PhlaEOTHRIPINAE}

Adrothrips intermedius (Bianchi, 1945)

Biologie : sur tiges vertes de diverses espèces de Casuarina.

Distribution: est de l'Australie, Nouvelle-Calédonie.

Matériel collecté: Nouméa (BIANCHI, 1945); 1 ô sur Casuarina, $20 \mathrm{~km}$ à l'ouest de Thio, 28.XII.1998.

Aleurodothrips fasciapennis (Franklin, 1908)*

Biologie : prédateur d'Aleyrodidae et Coccoidea.

Distribution : largement répandu dans les zones tropicales.

Matériel collecté: 29 sur feuilles de Psidium, 20 km à l'ouest de Thio, 28.XII.1998.

Athlibothrips caledonensis Bournier, 1993

Biologie : dans des cécidies constituées par des feuilles enroulées de Codia discolor (Cunoniaceae).

Distribution: Nouvelle-Calédonie.

Matériel collecté : mont Koghi (BOL'RNIER, 1993).

Bamboosiella cingulatus (Hood, 1919)*

Biologie: sur diverses plantes herbacées.

Distribution: largement répandu dans les zones tropicales.

Matériel collecté : $\varsubsetneqq$ et ơ sur herbes au bord de la route du Cap, $25 \mathrm{~km}$ au nord de Bourail. 22.XII.1998. 
Haplothrips angustus Hood, 1919

Biologie: sur fleurs de plantes herbacées.

Distribution: Australie, Nouvelle-Calédonie.

Matériel collecté : Nouméa et Népoui, Nouvelle-Calédonic (BIANCHI, 1945).

Haplothrips gowdeyi (Franklin, 1908)

Biologie : floricole, peut-être prédateur.

Distribution : largement répandu dans les zones tropicales.

Matériel collecté : Nouméa (BIANCHI, 1945);1 $\$ 10^{\dagger}$ sur fleurs de clémentinier, Pocquereux, 18.VIII. 1997 (leg. J.P. Deguine).

Haplothrips "victoriensis"; Bianchi, $1945=H$. victoriensis var. nepouiensis Bianchi, 1945

Matériel étudié: les spécimens identifiés de différents sites de Nouvelle-Calédonie par BIANCHI (1945) comme étant $H$. victoriensis, ont été réexaminés. Ce n'est pas cette espèce, mais pour le moment on ne peut l'identifier correctement; on observe en effet soit 1 cône, soit 2 cônes sensoriels sur l'article antennaire III, variation jamais enregistrée pour les espèces de ce genre. Les 2 femelles et le mâle syntypes de $H$. $v$. var. nepoziensis Bianchi ont été réexaminés; ils ont élé endommagés par l'éclaircissage au $\mathrm{KOH}$, mais sont probablement des individus dé petite taille de la même espèce. Il est possible que cette espèce soit endémique à la Nouvelle-Calédonie.

Haplothrips pallescens (Hood, 1919)*

Biologie: sur herbes diverses.

Distribution: sud-est asiatique, nord de l'Australie.

Matériel collecté: $q q$ et ô ơ sur végétation herbacée au bord de la route du Cap, $25 \mathrm{~km}$ au nord de Bourail. 22.XII.1998, et aे $10 \mathrm{~km}$ à l'ouest de Houailou, 26.XII.1998.

Karnyothrips flavipes (Jones, 1912)*

Biologie : prédateur de Coccoidea.

Distribution: largement répandu dans les zones tropicales.

Matériel collecté : 4 † sur branches mortes d'Acacia nilotica, $30 \mathrm{~km}$ à l'est de Pouembout, 23.XII.1998.

Karnyothrips melaleucus (Bagnall, 1911)*

Biologie : prédateur de Coccoidea.

Distribution : largement répandu dans les zones tropicales.

Matériel collecté : $q$ q sur végétation herbacée le long de la route du Cap, $25 \mathrm{~km}$ au nord de Bourail. 22.XII.1998.

Kellya milmani Girault, 1927

Biologie : dans de vieilles cévidies de diverses espèces d'Acacia atteintes de virescence, ex. : A. spirorhis. Distribution: est de l'Australie, Nouvelle-Calédonie.

Matériel collecté: Oundjo (BOURNIER, 1993).

Leeuwenia angulata Bianchi, 1952

Biologie : trés probablement phyllophage.

Distribution: Nouvelle-Calćdonie.

Matériel collecté: Saint-Louis (BIANCHI, 1952).

Macrophthalmothrips neocaledonensis Bournier, 1997

Biologie: mycophage sur branches mortes.

Distribution: Nouvelle-Calédonie.

Matériel colleclé: Mont-Nondoué (BOURNIER, 1997).

Neohoodiella grandisetis Bournier, 1997

Biologie: très certainement mycophage sur branches mortes.

Distribution: Nouvelle-Calédonie.

Matériel collecté: Rivière-Bleue (BOURNIER, 1997).

Podothrips xanthopus Hood, 1919

Biologie : prédateur de Coccoidea sur graminées.

Distribution: est de l'Australie, Nouvelle-Calédonie.

Matériel collecté: Nouméa (BIANCHI, 1945). 
Stephanothrips occidentalis Hood \& Williams, 1925

Biologie : mycophage sur feuilles et branches mortes.

Distribution: largement répandu dans les zones tropicales.

Matériel collecté : Pindai et Rivière-Bleue (BOURNIER, 1997).

Teuchothrips cleistanthi (Bournier, 1975), comb. $\mathbf{n}$.

Décrite comme Hoplothrips (Odontoplothrips) cleistanthi, cette espèce présente 1 cône sensoriel sur l'article antennaire III, et 3 sur l'article IV, caractère typique de genres comme Teuchothrips et liothrips.

Biologie : dans des galles en forme de bourse sur feuilles de Cleistanthus sp. (Euphorbiaceae).

Distribution: Nouvelle-Calédonie.

Matériel collecté : Koumac, Palachidji (BOURNIER, 1975).

Teuchothrips kraussi (Bournier, 1993), comb. n.

Décrite comme Oncothrips kraussi, cette espèce présente, sur l'article antennaire IV, 3 cônes sensoriels au lieu de 2 comme observé chez les espèces du genre Oncothrips ; elle fait partie du complexe Teuchothrips qui comprend de nombreuses espèces en Australie, et qui nécessitera une révision approfondie.

Biologie : vivant dans les cécidies formées par des feuilles enroulées.

Distribution: Nouvelle-Calédonie.

Matériel collecté : Mont-Koghi (BOURNIER, 1993).

Teuchothrips noumeaensis Bournier, 1993

Biologie : vivant dans les cécidies formées de feuilles enroulées d'Hibbertia tontoutensi (Dilleniaceae). Distribution: Nouvelle-Calédonie.

Matériel collecté : vallée de la Tontouta (BOURNIER, 1993).

Teuchothrips ornatus Bournier, 1993

Biologie : vivant dans les cécidies formées de feuilles enroulées d'Hibbertia lucens (Dilleniaceae). Distribution: Nouvelle-Calédonie.

Matériel collecté : Mont-Koghi (BouRNIER, 1993).

Teuchothrips pacificus (Bianchi, 1952), comb. n.

Décrite comme Arrhenothrips pacificus, cette nouvelle combinaison est basée sur la comparaison avec les spécimens de Teuchothrips originaires de Nouvelle-Zélande et d'Australie.

Biologie: dans les cécidies formées de feuilles enroulées.

Distribution: Nouvelle-Calédonie.

Matériel collecté : Bois-du-Sud (BIANCHI, 1952).

\section{AUTEURS CITÉS}

BAGNALL R. S., 1908. - On some new and curious Thysanoptera (Tubulifera) from Papua. Annals and Magazine of Natural History, 8: 355-363.

- 1928.- On some Samoan and Tongan Thysanoptera, with special references to Ficus gall-causers and their inquilines. Thysanoptera. In: Insects of Samoa, 7: 55-76. London: British Museum (Natural History).

BIANCHI F.A., 1945. - Introduction to the Thysanoptera of New-Caledonia. Proceedings of the Hawaiian Entomological Society, 12 : 249- 278.

1952.- Additions to the Thysanoptera of New-Caledonia. Proceedings of the Hawaiian Entomological Society, $14: 385-390$.

BOURNIER A., 1975. - Hoplothrips (Odontoplothrips) cleistanthi n.sp. Thysanoptère cécidogène de Nouvelle-Calédonie. Marcellia, 38: 323-326.

— 1993.- Thysanoptères gallicoles de Nouvelle-Calédonie. Bulletin de la Société entomologique de France, 98: 357-366.

BOURNIER J.-P., 1997. - Thysanoptères des forêts primaires de Nouvelle-Calédonie. I. Annales de la Société entomologique de France, 33 : 139-153.

CHAZEAU J., 1995. - Bibliographie indexée de la faune terrestre de Nouvelle-Calédonie. Systématique, écologie et biogéographie. ORSTOM, Paris, 95 p.

CoHis F., 1953. - Un dangereux parasite du glaïeul introduit récemment en Nouvelle-Calédonie (Taeniothrips simplex Morison). Revue Agriculture de Nouvelle-Calédonie, 11-12 : 18-21. 
GUII. AUMIN A.. 1948. - Flore analytique et synoptique de la Nonelle-calédonite. (ORSTON. Paris. 369 p.

Motition D., 1939. - Thysanoptera collected by the Mangarevan expedition. Occasional Petpers of the Bishop Museum. 15: 141-148.

1940. - Thysanoptera from New-(iuinea and New-Britain. Occasional Papers of the Bishop) Museum. 15: 243-270.

1944. Thysanoptera of Fiji. Occasional Papers of the Bishop Muscum. $17: 267-311$.

1947. . Thysanoptera from Vew-(juinea, the Philippine Islands and the Malay Peninsular. P'an-

Pacific Entomologist, 23 : 172-180.

Moditon D. \& Stianweiden J.B., 1932. New Marquesan Thysanoptera. Pacific entomological Survey, 1/17: 165-168.

1933. Thysanoptera from the Society Islands. Pacific entomological Survel: 66: 29.33.

Mound L.A., 1970. - Thysanoptera from the Solomon Islands. Bulletin of the British Mhasem (Natural History). Entomology, 24:83-126.

- 1996. - Thysanoptera. p. 249-332, 397-414 (Index), in Wells A.. Zoological ('ataloglic of Australia. Volume 26. Psocoptera, Phthiraptera, Thysanoptera. Melboume ('siro Australia. 1999 a. - Thysanoptera from lord Howe Island. Australian Fntomologist, 25: 11:3-120.

1999 b. - Saltatorial leaf-feeding Thysanoptera (Thripidae. Dendrothripinae) in Australia and New-Caledonia, with newly recorded pests of ferns. ligs and mulberries. Australian Journal of Entomology, $38: 257-273$.

Motnd L. A. \& WALKER A.K., 1982. - Terebrantia (Insecta: Thysanoptera). Fanna of New Zealand. $1: 1-113$.

1986. - Tubulifera (Insecta: Thysanoptera). Fauna of New Kealand. 10:1-140.

1987. - Thysanoptera as tropical tramps: new records from New /ealand and the Pacitic. Wen Zealand Entomologist. 9: 70-85.

SAKIMURA K.. 1969. New species of subgenera Isothrips and Isochuetothrips from (Ocania and Australia. with note on changes in nomenclature (Thysanoptera. Thripidae). Pacific Insects, 11 : 71-80.

Michel BOULARD (EPHE et MNHN. l'aris)

Métamorphoses et Transformations animales.

Oblitérations evolutives. Paris, editions Boubéc, avril 2000. ISBN 2-850(04-092-4. Prix:190 F en librairic*. Un volume au format $16 \times 24 \mathrm{~cm}$. broché sous jaquette illustrée. de 311 pages. 49 ligures dans le texte, 7 pl. en sépia et $16 \mathrm{pl}$. hors texte en couleurs.

L'ouvrage de Michel Boulard est une synthèse richement illustrée sur la partie post-embryogénique du développement animal. Il précise:

- les concepts de métamorphose et de transformations morphologiques - souvent confondus, mais que l'auteur révise et redéfinit de manière précise - et celui, nouveaul. des obliterations morphogénitiques:

- l'histoire des découvertes qui ont conduit à établir la postembryogénic;

les caractéristiques et les modalités des métamorphoses et des transformations animales. plus ou moins développés suivant les groupes de métazoaires considérés:

le vocabulaire propre à cette partic de la biologie animale : - une bibliographie riche de 140 ouvrages et 369 articles.

"Synthese et conclusion remarguables. Autrefois, on aurait appelé le livre de Michel Boulard Philosophie des Mélamorphoses de la même façon que Lamarck appela Philosophice zoologicue l'ouvrage fondamental que nous connaissons tous aujourd hui." (Michel Delsol)

* $180 \mathrm{~F}$. franco de port pour toute ccmmande a : I aboratoire de Biologie et Évolution des Insectes. L:Pllt: 45. rue Buffon. 75005 Paris (e-mail : boulard amnhn.fr)
Vient de paraître (avril 2000)

\section{Métamorphoses et TRANSFORMATIONS ANIMALES}

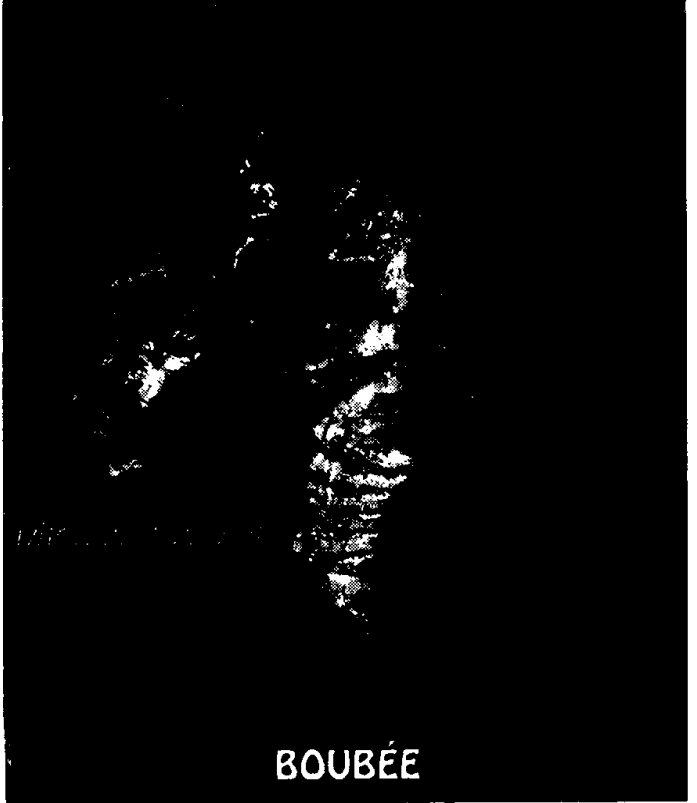

